\title{
A Development of Computer-Aided Diagnosis System using Fundus Images
}

\author{
Jun-ichiro HAYASHI ${ }^{+}$, Takamitsu KUNIEDA*, Joshua COLE ${ }^{+}$, Ryusuke SOGA \\ Yuji HATANAKA ${ }^{\#}$, Miao Lu\#, Takeshi HARA ${ }^{\#}$ and Hiroshi FUJITA ${ }^{\#}$ \\ +:Softopia Japan, 4-1-7 Kagano, Ogaki-city, 503-8569 JAPAN \\ *:TAK Co.,LTD., 4-35-12 Kono, Ogaki-city, 503-0803 JAPAN \\ \#:Gifu University, 1-1 Yanagido, Gifu-city, 501-1193 JAPAN
}

\begin{abstract}
We developed a computer-aided diagnosis $(C A D)$ system. The purpose of this project is to aid physicians in diagnosing medical images. A CAD system can help physicians by displaying useful information such as the location of abnormalities. Although many kinds of images are used in medical diagnosis, we have focused our research to methods of analyzing fundus images. Physicians normally observe the condition of blood vessels and the retina when examining fundus images. Therefore, we are researching analysis schemes for these objects.

Presently, we have improved schemes to detect abnormalities on the retina and analyze blood vessel intersections. In addition, we have developed methods of detecting abnormal widths in blood vessels. We have collected about 450 fundus images and used them to test our schemes. We have obtained relatively good results.

In this paper, we give an outline of our system and describe each processing step.
\end{abstract}

\section{Introduction}

In recent years, Japan and other parts of the world have seen the development of an aging society and an increase in lifestyle-related illnesses. As a result, more and more emphasis is being placed on conducting routine physicals and thorough medical examinations to detect diseases early. This is a huge task for medical institutions since the exams must be conducted regularly, in some cases at specialized facilities. Medical image diagnosis is extremely useful in determining signs of disease and is relatively less demanding on the examiner. Therefore, it has been the focus of much research. This has led to some remarkable technological advances. As a result, exams using medical imaging devices such as CT and MRI are becoming as widely used as traditional X-ray exams. Consequently, the number of regions that are scanned has increased to meet the objectives of each exam. Usually a physician is in charge of diagnosing these medical images, but in most cases, this is not a specialized position. The physician normally has other duties to perform. As a result, the burden placed on physicians diagnosing these images is by no means light, especially when these types of routine exams are becoming more and more common due to the societal factors mentioned above.

Various approaches to lessen the burden of these physicians have been considered. One of them involves using computers to assist in processing the images. For computers, image processing is extremely demanding on the processor and poses many problems with respect to processing speed. However, these problems have been gradually solved due to the rapid development of information technology today. Therefore, technical applications of computer image processing have become common in many fields. In medical image diagnosis, 
specialized systems are being developed that can analyze specific types of images almost as well as physicians. With the effective use of these systems, in the future it will be possible to lessen the workload of physicians as well as improve diagnostic accuracy by reducing medical oversights.

In our research, we developed a computer-aided diagnosis system using fundus images. Through fundus images, it is possible to determine not only diseases of the eye but also those whose symptoms are evident in blood vessels. For example, physicians determine illnesses from abnormalities of the retina, such as white spots and hemorrhaging, and the appearance of vascular intersections. We have focused our research on this type of analysis processing. Although we were able to detect the abnormal regions, the results also yielded many incorrect detections. Therefore, in order to develop a more practical detection technique, we have decided to examine not only abnormal cases but normal cases as well. In this way, we hope to achieve a better method of detecting abnormal regions.

In this paper, we give an overview of our system and image processing. In Chapter 2, we explain the diagnosis of fundus images. In Chapter 3, we describe the method of extracting unusable fundus images from the test data. In Chapter 4, we describe the detection of abnormalities in the retina and ways of improving the detection rate. In Chapter 5, we describe the processing of main blood vessels, ways of improving this process, and the results. Finally, in Chapter 6, we state our conclusion and outline our future work.

\section{Analyzing fundus images}

\subsection{Characteristics of fundus images and their analysis}

Fundus diagnosis consists of inspecting the back of the eyeball as seen from the front of the face. This region is called the fundus of the eye and predominately consists of the retina. In fundus diagnosis, a photograph of the region is usually taken and examined. As shown in the cross-section of the eyeball depicted in Figure 1, the hemispherically shaped retina appears as a planar surface in the fundus image. Physicians need to be aware of this fact when examining the images. The retina is a part of the body in which blood vessels may be observed directly. It is useful not only for detecting abnormalities of the eye but also in discovering blood vessel abnormalities. As a result, the main purpose of fundus diagnosis is the early detection of diseases whose symptoms are evident in the body's blood vessels.

In our research, we observed the characteristics of fundus images and used computer image analysis to detect blood vessel abnormalities. The chief abnormalities visible in fundus images may be divided into two groups: diseases of the eye, such as glaucoma and cataracts, and lifestyle-related illnesses, such as hypertension, arteriosclerosis, and diabetes. The occurrence of lifestyle-related illnesses is determined by factors such as the state of the blood vessels or evidence of hemorrhaging in the fundus image.

\subsection{Characteristics of the input image}

The input images used in our research were taken mainly from $35 \mathrm{~mm}$ positive film and were digitized using a commercial flatbed-type scanner. The digitized pictures are 8-bit RGB with $1600 x 1600$ resolution. Figure 2 shows an example of the input image.

\section{Eliminating unusable images from the test data 3.1. Method of elimination}

Using the following criteria, we eliminated unusable fundus images from the test data. 
In these cases, the images were either of too poor a quality to be used for diagnosis or showed signs of a serious medical problem, thus requiring a thorough check by a physician.

- Fuzzy image (image out of focus, cataracts, etc.).

- Hard to determine retinal structure (existence of very large abnormality, etc.).

In order to eliminate these types of images, we used the following methods to identify them.

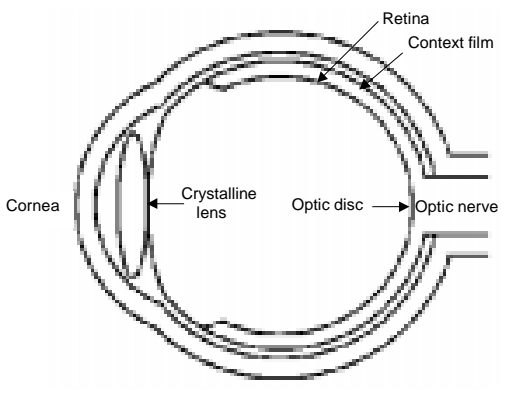

Fig. 1. Cross-section of the eyeball.

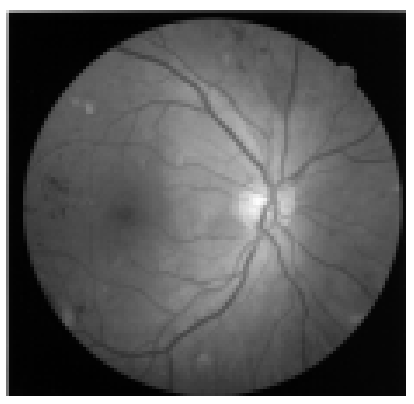

Fig. 2. Sample input image.

Step 1: Extract the edges of the retina. If the number of edge pixels is remarkably low, the image is fuzzy.

Step 2: Convert the image to monochrome, perform binarization, and detect abnormalities on the retina. Repeat this process on an inverted monochrome image. If a large number of abnormalities are detected, the image is not suitable for analysis by this system.

\subsection{Experiment}

We experimented on 246 images taken from mass screenings. Nine images were eliminated from this group. The results are shown in Table 1.

Figure 3 shows examples of correct eliminations.

Table 1. Results of eliminating unusable images.

\begin{tabular}{|c||c|c|c|}
\hline Image & No. of True Positives & No. of False Positives & Total \\
\hline \hline Fuzzy & 1 & 1 & 2 \\
\hline Contains large no. of abnormalities & 5 & 2 & 7 \\
\hline Total & 6 & 3 & 9 \\
\hline
\end{tabular}
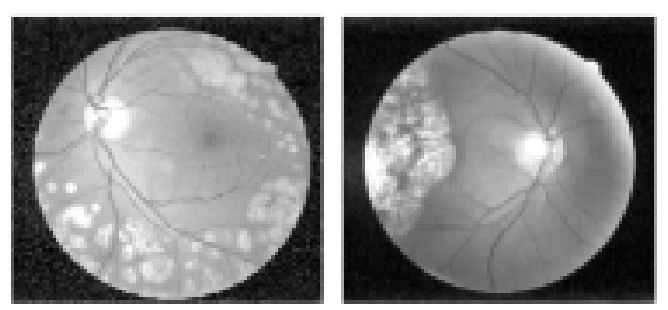

Fig. 3. Examples of eliminated images. 


\subsection{Results}

Using the present method of elimination, some images of slightly low contrast were falsely identified as fuzzy. In addition, some images having clearly visible vascular membranes were falsely identified as having a large abnormality. However, even though these images were falsely identified, they are nevertheless worthy of elimination by the system. In the first case, the low contrast makes the retinal features indistinguishable. In the second case, noise makes the retinal features indistinguishable. It is necessary for us to reevaluate our elimination criteria to include these cases. Looking at these results, we believe that our method is useful.

\section{Method of detecting abnormalities on the retina}

\subsection{Abnormalities on the retina}

We learned from physicians that abnormalities on the retina take two major forms: hemorrhaging and white spots. At first, our purpose was to detect only these two phenomena. However, many kinds of abnormalities may exist on the retina. As a result, these phenomena were falsely determined to be cases of hemorrhaging or white spots. Since detecting these types of phenomena is useful from a medical standpoint, we decided to broaden our definition of abnormalities to include "red" (shown as black areas on a monochrome image) and "white" (shown as white areas on a monochrome image) abnormalities. In this section, we describe our method of detecting these abnormalities.

\subsection{Method of detecting red abnormalities on the retina}

4.2.1. Problems in previous method: Figure 4 shows the process used in our previous method. We experienced some problems with this method.

\begin{tabular}{|l|}
\hline Step 1: Convert color image to monochrome. \\
\hline Step 2: Extract areas of low density using binarization. \\
\hline Step 3: Delete vascular region. \\
\hline Step 4: Delete unnecessary elements. \\
\hline
\end{tabular}

Fig. 4. Previous method of detecting abnormalities.

[Problem 1]: Falsely determining a section of blood vessel as an abnormality.

Relatively thin vessels appear to be segmented and therefore are detected as separate objects during vascular detection.

[Problem 2]: If an abnormality touches or overlaps a blood vessel, it is determined to be a blood vessel.

Under the previous method, if an abnormality touched or overlapped a blood vessel, it was determined to be part of the blood vessel and deleted from the list of abnormalities.

[Problem 3]: If the background (e.g., blood vessels in the vascular membrane) is clearly visible, the number of misdetections increases.

There is a layer of the retina called the vascular membrane. If it is clearly visible in the image, it is difficult to determine the blood vessels in the retina.

[Problem 4]: Misdetections caused by spots in the background

In our previous method, we defined an abnormal region as one in which a difference in density exists between a given pixel and the average pixel value of its neighbors. However, 
this only takes the density value of each pixel into consideration. It does not determine regions of similar density values. As a result, tiny spots yield misdetections.

4.2.2. The improved method: We made the following improvements to correct the problems of our previous method.

[Improvements for Problem 1]:

In order to decrease the amount of segmentation that appear in thin blood vessels, we drew a line down the center of each blood vessel. If an abnormal candidate region overlaps this line, it was determined to be a misdetection. Figure 5 shows the process of making this centerline.

Step 1: Extract blood vessel candidate region from monochrome image using binarization.

Step 2: Delete unnecessary minute regions.

Step 3: Expand region.

Step 4: Thin region.

Step 5: Expand region.

The process above creates a centerline that is 3 pixels wide. If an abnormal candidate region overlaps with a blood vessel, it is determined to be part of a region forming a blood vessel and therefore a misdetection.

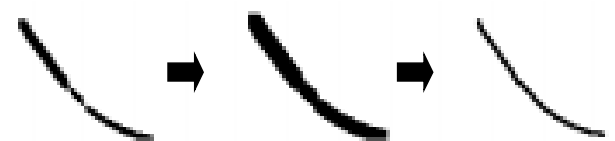

Fig. 5. Rejoining a segmented blood vessel.

[Improvements for Problem 2]:

The following method was examined in order to detect abnormalities that touch the blood vessel during the binarization stage (as shown in Figure 6).

Step 1: Contract regions in binarized image.

Step 2: Determine contiguous regions of a large area to be blood vessels and delete them.

Step 3: Process remaining regions using the conventional elimination method for unnecessary regions.

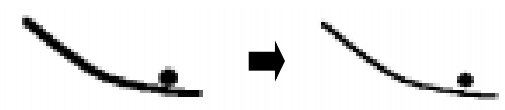

Fig. 6. Separating an abnormality from a blood vessel.

[Improvements for Problem 3]:

We analyzed the density and color distribution of the retina. Our results indicated that the density difference is large in the blood vessels of the vascular membrane. Then we narrowed the width of the brightness value of the whole image, lessened the influence of contrast, and raised the detection accuracy using differences in color.

Step 1: Convert RGB color image to HSV color image.

Step 2: From HSV color image, calculate value (V) histogram and narrow gradient width.

Step 3: Convert HSV color image to RGB color image. 
[Improvements for Problem 4]:

We added steps to determine abnormalities by calculating the average density of each abnormal candidate region and those of its neighbors. This reduces the influence caused by the image background.

Step 1: Calculate position of each abnormal region and define the area that contains it.

Step 2: Calculate the average density of each region and those of its neighbors.

Step 3: If the difference is less than the threshold, it is declared a misdetection.

4.2.3. Experiment: We experimented on 230 images, 17 of which contained red abnormalities. The results are shown in Table 2. Figure 7 shows an example of true positive.

Table 2. Results of the improved method.

\begin{tabular}{|c||c|c|c|}
\hline & No. of True Positives & No. of False Positives & No. of Oversights \\
\hline \hline Results & 10 & 40 & 7 \\
\hline
\end{tabular}

50 abnormalities were detected in 230 images.

4.2.4. Results: Of the cases that could not be detected by the improved method, we paid particular attention to those that overlooked instances of hemorrhaging. There were 2 main patterns.

- Hemorrhaging in thin blood vessels (as shown in Figure 8-a)

Thin blood vessels are not considered abnormalities since we do not want them to be falsely detected.

- Overlapping of numerous blood vessels (as shown in Figure 8-b)

The boundaries between blood vessels could not be determined.

We believe these errors were based on the initial conditions of the step that removes unnecessary regions since the red and white regions were divided during binarization. In addition, when analyzing the misdetections, we found many instances of thin blood vessel edges and vascular intersections. Therefore, further improvements are necessary.
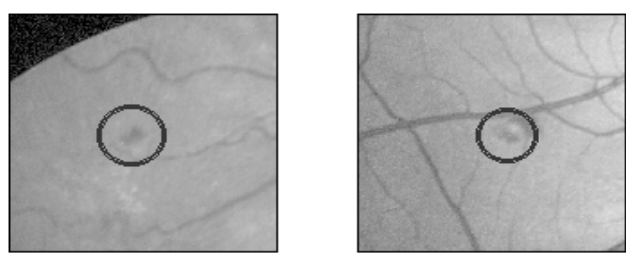

Fig. 7. Examples of true positives.

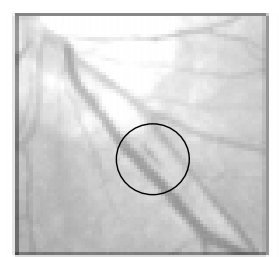

(a)

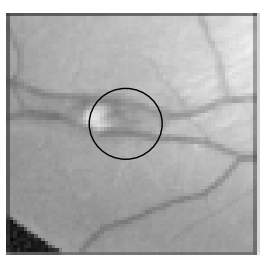

(b)

Fig.8. Examples of false negatives.

\subsection{Method of detecting white abnormalities in the retina}

4.3.1. Problems in previous method: By taking the negative of the monochrome image, it is possible to detect white abnormalities in the same way as red ones. However, there were some huge differences in the way the two detection methods yielded misdetections. Unlike detecting red abnormalities, there were no problems with falsely detecting sections of blood vessels, but the following problems did occur.

[Problem 1]: Misdetections around the optic disc. 
Like red abnormalities and blood vessels, since white abnormalities and the optic disc are of the same color, it is hard to distinguish them by simply performing binarization on the monochrome images. It is possible to distinguish the two when the optic disc appears as a solid region. However, the optic disc cannot be distinguished from white abnormalities when it appears fragmented.

[Problem 2]: Misdetections near blood vessels.

Misdetections occurred in the small areas between large blood vessels in the optic disc. This was due to the large difference in density occurring near the optic disc.

4.3.2. The improved method: We made the following improvements to resolve the problems above.

[Improvements for Problem 1]:

We decided not to perform the detection process on the area surrounding the optic disc. The new process is as follows.

Step 1: Extract the optic disc and define a mask in the circular region containing it.

Step 2: Delete abnormalities inside the mask.

[Improvements for Problem 2]:

We decided to compare the pixels surrounding the blood vessels in order to determine whether the candidate region is valid. The process is as follows.

Step 1: Generate an image consisting solely of large blood vessels and define it as a mask.

Step 2: Calculate the difference between the average densities of the candidate and surrounding regions. Remove the candidate region from consideration if the difference is less than a given threshold. Use the mask made in the previous step to calculate the average density of the surrounding region, but do not consider the blood vessels when making the calculation.

4.3.3. Experiment: We experimented on 246 images of which 30 images contained white abnormalities. The results are shown in Table 3. Figure 9 shows an example of a true positive.

Table 3. Results of the improved method.

\begin{tabular}{|c||c|c|c|}
\hline & No. of True Positives & No. of False Positives & No. of Oversights \\
\hline \hline Results & 21 & 38 & 9 \\
\hline
\end{tabular}

4.3.4. Results: It is necessary to reexamine this method since many misdetections occurred in areas surrounded by multiple blood vessels. The misdetections around the optic disc were mainly resolved; however, misdetections did occur in the rare event that the optic disc was not clearly visible. In addition, oversights occurred in small regions. We believe we can fix this by revising the conditions used to limit the candidate regions. Despite the insufficient number of test images, we achieved a relatively good detection rate for white spots (as shown in Figure 9). Oversights consisted of abnormalities of little medical significance.

\section{Analyzing main blood vessels}

\subsection{Analyzing blood vessels}

Until now we have examined many methods of analyzing blood vessels. In this research, we focused on two points. 


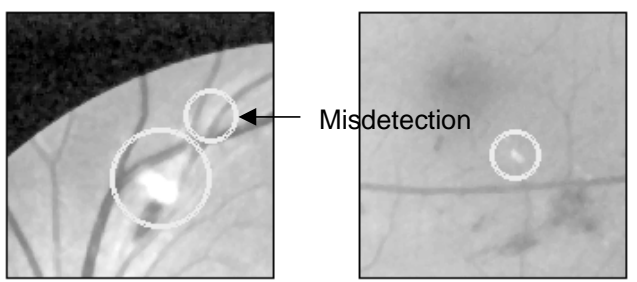

Fig. 9. Detecting white abnormalities.

The first is to analyze vascular intersections. A phenomenon occurs at the point where an artery and vein cross. The artery presses down on the vein, squeezing it and causing it to appear deformed. Figure 10 shows the most common forms. This phenomenon can be extremely useful in determining the existence and severity of arteriosclerosis.

The second is to analyze abnormal vascular widths. Normally the width of a blood vessel does not vary drastically. However, in cases of high blood pressure or arteriosclerosis, such variability may be present; therefore, it is important from a medical standpoint to note these cases.

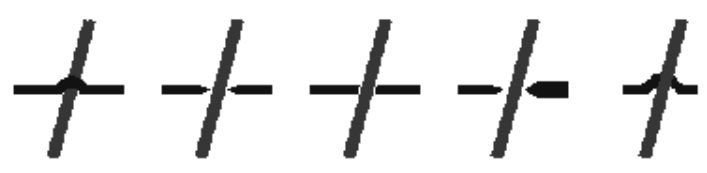

Fig. 10. Examples of vascular intersections.

\subsection{Analyzing vascular intersections}

We proposed the use of a band filter. It allows us to detect vascular intersections with relatively few oversights and good results, even among cases in which part of a blood vessel was not clearly visible. However, when applied to a larger set of test images, it yielded more misdetections. The clarity of the blood vessels seems to have little effect on the number of misdetections; however, the greater the concentration of blood vessels, the greater the number of misdetections. We believe that this is because the probability that a region will be considered as an intersection will become greater as the number of blood vessels passing though the candidate region increases. Therefore, we attempted to reduce the number of misdetected vascular intersections by reducing the number of misdetected regions that are not vascular intersections.

5.2.1. The improved method: Our previous method is based on the concept that if two blood vessels cross inside a given region, the vessels will cross the boundary of the region at a minimum of four places. But this condition is satisfied not only in the case of vascular intersections, but also when numerous blood vessels exist in the region. Therefore, we decided to revise our set of conditions by analyzing the misdetections and abstracting the resulting pattern. Figure 11 shows examples of misdetected vascular intersections.

5.2.2. Experiment: We experimented on 100 images to determine whether the point of vascular intersection could be detected precisely. The results revealed that 191 out of 758 detected intersections were false, yielding an error ratio of $25.1 \%$. In other words, 1.9 out of 7.5 intersection points in a given image were erroneous. No problems arose in detecting vascular intersections by changing our set of conditions, since the actual method of detecting them did not change. 

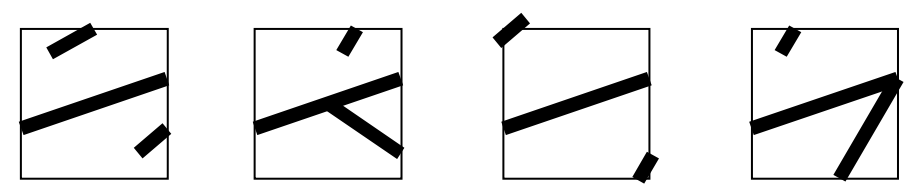

Fig. 11. Examples of misdetected vascular intersections.

5.2.3. Results: The number of misdetections tends to increase in images that contain many narrow blood vessels. These kinds of images were present in the test data set. However, by improving the vascular intersection detection method, we achieved relatively stable results overall. We must examine the improved method further in order to reduce the number of misdetections and improve the detection accuracy.

\subsection{Analyzing vascular width}

5.3.1. Variations in vascular width: Normally vascular width does not vary drastically, except at junction points or intersections. If it does, it usually implies that there is a problem worthy of medical attention. However, it is difficult to strictly measure the exact change in width, so most physicians rely on their experience or intuition to make judgments. Therefore, we examined methods of measuring vascular width quantitatively.

5.3.2. Procedure: The procedure is as follows.

\section{Step 1: Pre-processing}

Using our method of detecting blood vessels, we extract the blood vessels from the input image, removing the smaller ones. Then, using the extracted image, we search for junction points and intersections and segmented each blood vessel at these points. When segmenting each blood vessel, we do not include the neighboring pixels around each junction point and intersection. We also remove any noticeably small blood vessels from consideration.

Then we perform steps 2 and 3 on each blood vessel segment.

Step 2: Measuring vascular width

Perform thinning on the blood vessel. Sequentially calculate the width along each pixellength section of the blood vessel segment and store the results in an array. In order to measure the width along each section, approximate the centerline of the section and calculate its perpendicular. Count the number of pixels within the blood vessel that reside along the perpendicular and declare the result to be the sectional width.

Step 3: Detecting abnormal sections

Calculate the average vascular width of each blood vessel based on the results of step 2 . Compare this to the actual width of every section along each blood vessel. If a section is narrower than a given threshold, declare it an abnormal section.

Step 4: Determining the results

After completing steps 2 and 3 on each blood vessel, count the number of abnormal blood vessels, mark each abnormal section, and declare these to be the detection results.

5.3.3. Experiment: We experimented on 100 images, which included 11 images having blood vessels with abnormal widths.

We decided to use the number of segmented blood vessels as the unit of measurement. Table 4 shows the results. 
Table 4. Results of this method

\begin{tabular}{|c||c|c|c|}
\hline & No. of Detected Segments & No. of Total Segments & Detection Rate \\
\hline \hline Abnormal & 13 & 17 & $76.5 \%$ \\
\hline Normal & 764 & 945 & $80.8 \%$ \\
\hline
\end{tabular}

5.3.4. Results: We showed the results of this method to a physician. When we showed him blood vessels that he declared normal, but the system declared abnormal, he reversed his decision. Since physicians often base their judgment of abnormal vascular widths on intuition and personal experience, they occasionally make errors. In light of this, we believe that having a quantitative method of measuring vascular widths is one of the strengths of our system.

\section{Conclusion and future works}

We developed a computer-aided diagnosis system for fundus images. In this paper, we have explained how each feature works. Figure 13 shows the system interface. After revising our methods of analysis and performing experiments on a test data set, we were able to reduce the number of misdetections. However, we do not have sufficient examples of abnormal cases.

Our future goal is to gather more data on which to experiment. We would also like to improve our detection rate further.

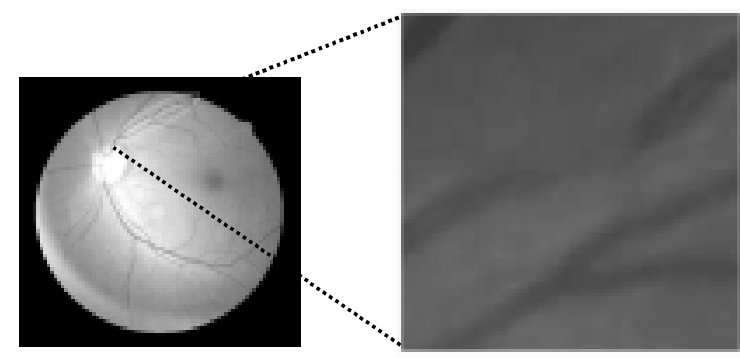

Fig. 12. Detected blood vessel

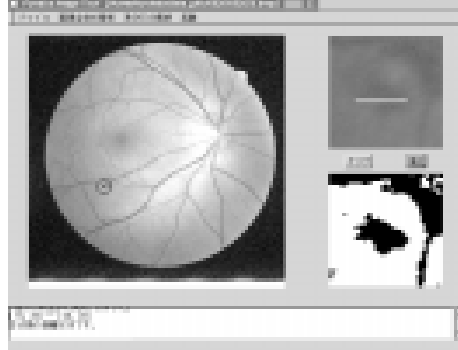

Fig. 13. System interface

\section{Acknowledgements}

The authors wish to express their thanks to those who have supported this project. In particular, we would like to thank the Department of Medicine at Gifu University, for lending their support.

\section{References}

[1] Kensaku MORI, Akihiro KUSHIDA, Jun-ichi HASEGAWA, Yasuhito SUENAGA, Jun-ichiro TORIWAKI, and Kazuhiro KATADA, A Method for Generating a Virtually Stretched Image of the Tract Basing upon Deformation of a 3-D Medical Image with Application to Stomach X-Ray CT Images, Trans. of IEICE, D-II, Vol. J83-D-II, No. 1, pp.351-361 (Jan. 2000).

[2] Yuji UKAI, Noboru NIKI, Hitoshi Satoh, and Shigeru WATANABE, Computer Assisted Diagnosis System for Coronary Calcifications Based on Helical CT Images, Trans. of IEICE, Vol. J83-D-II, No. 1, pp.342-350 (Jan. 2000).

[3] Jun-ichiro TORIWAKI, Trends and Future Problems in Computer Aided Diagnosis X-ray Images, Trans. of IEICE, D-II, Vol. J83-D-II, No. 1, pp.3-26 (Jan. 2000).

[4] Marc Lalonde, Lang Gagnon and Maric-Carole Boucher, Non-recursive paired tracking for vessel extraction from retinal images, Proc. of VI2000, pp. 61-68 (May 2000).

[5] KAZUAKI SUGIO, TAKAMITSU KUNIEDA, HIROSHI FUJITA, TAKESHI HARA, TAKESHI KAWASE, KAZUMI OGAWA, AKIRA ISHIDA, and MICHIHIRO INAGAKI, Analysis of Blood Vessel Intersections in Fundus Images, Japanese Society of Radiological Technology, Vol. 56 No.3, pp.507-509 (Mar. 2000). 